\title{
Mars' Ancient Dynamo and Crustal Remanent Magnetism
}

A whitepaper submitted to the 2021 Planetary Science Decadal Survey

Primary author:

Mittelholz, Anna University of British Columbia, Vancouver, Canada

(604-446-0634; amittelh@eoas.ubc.ca)

\section{Co-authors:}

Connerney, J. Goddard Space Flight Center, USA

Espley, J. Goddard Space Flight Center, USA

$\mathrm{Fu}, \mathrm{R}$. Harvard University, USA

Johnson, C. L. University of British Columbia, Canada; Planetary Science Institute, USA

Langlais, B. $\quad$ CNRS/University of Nantes/University of Angers, France

Lillis, R. J. $\quad$ University of California Berkeley, USA

Morschhauser, A. GFZ Potsdam, Germany

Ravat, D. University of Kentucky, USA

Vervelidou, F. Massachusetts Institute of Technology, USA

Volk, M. Harvard University, USA

Weiss, B. P. Massachusetts Institute of Technology, USA 
Executive Summary. Mars Global Surveyor (MGS) confirmed the lack of a present-day core dynamo field on Mars, but also detected surprisingly strong crustal magnetic fields. Combined with remanent magnetization in martian meteorites, this indicates that ancient Mars generated a dynamo field. Expanding our understanding of crustal magnetism on Mars will elucidate the characteristics and timing of the ancient dynamo, as well as (near-)surface processes that have modified crustal magnetic fields throughout time including impacts, water interactions (fluvial and hydrothermal), volcanism and tectonics. Furthermore, the existence, strength, and nature of a global magnetic field have profound effects on atmospheric escape. First, in the absence of a global magnetic field, ion outflow can occur all over the planet. However, strong electric fields that occur in the polar regions of intrinsic magnetospheres could possibly result in more atmospheric loss globally than the non-magnetized case ${ }^{1}$. Second, sputtering escape, a major contributor to early Mars atmospheric loss ${ }^{2}$, would have been almost completely inhibited when Mars had a global dipole field. For these reasons, the history of the martian dynamo is inextricably linked with the evolution of Martian climate and habitability. Furthermore, crustal fields influence the distribution of harmful radiation reaching the surface, a major consideration for future human exploration. Thus, the martian crustal magnetic field holds broad implications for Mars' early habitability, interior structure, thermal history, and for the fundamental physics of planetary dynamos, as well as human exploration. However, we have almost no information on crustal magnetism at scales smaller than $100 \mathrm{~km}$. The main goal of this white paper is to motivate high-precision, high resolution regional mapping and local investigations of crustal magnetic fields and magnetization on Mars.

Our primary recommendation is for NASA to focus on planning and implementation of magnetometers on aerial platforms such as airplanes, drones and/or long-lived balloons to obtain low-altitude magnetic measurements over kilometers to hundreds of kilometers.

Value. Determining the nature, origin and age of Mars' crustal remanent magnetization will provide valuable knowledge pertinent to high priority topics in Mars science:

1) The formation and evolution of the crust, including its mineralogy and modification over the past $\sim 4.5 \mathrm{Gyr}$, by tectonic, impact, fluvial, hydrothermal and magmatic processes (MEPAG Goal III $A+B$ )

2) The evolution of the core dynamo, and its implications for core composition and dynamics, interior evolution including early global heat flow, mantle dynamics and tectonic regime (e.g., whether Mars had an early phase of plate tectonics) (MEPAG Goal III B)

3) The link between atmosphere evolution and the extinction of the martian dynamo and thus important information on habitability (MEPAG Goal II C)

4) Implications for future human exploration (MEPAG Goal IV A)

Current knowledge of Mars crustal magnetism. In this section, we summarize how existing datasets have contributed to our current understanding of the Martian crustal magnetic field and the broader implications for the planet's evolution as well as limitations associated with the respective data sets.

Satellites - (a) Coverage and models: Magnetic field data from the MGS mission confirmed that Mars does not have a present-day global magnetic field ${ }^{3,4}$. However, strong crustal magnetic 
fields, concentrated in the Southern hemisphere, provide evidence for rocks magnetized by an ancient dynamo. MGS data, in particular those collected on the nightside (i.e., less contaminated by external magnetic fields) allowed construction of crustal magnetic field models ${ }^{5-8}$.

The MGS magnetometer data set is densely sampled at mapping orbit altitudes of $370-430 \mathrm{~km}$ ( $\sim 30,000$ orbits), while it is very sparsely sampled at lower altitudes (a few hundred elliptical and mainly dayside orbits at $90-170 \mathrm{~km}$ from the pre-mapping phase; e.g. ${ }^{6,7}$ ). The MGS electron reflectometer data set allowed remote sensing of the field intensity at lower altitudes down to $180 \mathrm{~km}$, but did not provide global coverage ${ }^{9}$. Since the last decadal survey (2014-present), the MAVEN magnetometer has provided global coverage at altitudes as low as $\sim 135 \mathrm{~km}{ }^{10}$. Models including this dataset provide the most up-to-date and highest resolution global view of the crustal magnetic field, as shown in Figure $1^{11,12}$.

Satellites - (b) Global distribution of crustal fields and the dynamo: Crustal fields are mostly concentrated in the heavily-cratered southern hemisphere, with a sparse pattern of mostly weak fields in the north. The source of this hemispheric difference has been variously interpreted as result of a single hemisphere dynamo ${ }^{13}$, differences in mineralogy ${ }^{14}$, or in crustal thickness ${ }^{15}$. Any of these scenarios has major implications for the present state and history of Mars.

Current data indicate an ancient global martian magnetic field, prompting the questions of when and for how long did such a core dynamo operate? The answer to these questions has important implications for the evolution of Mars' thermal and atmospheric state. Orbital data allow correlations of magnetic field signatures (or inferred magnetizations) with geological features that can be associated with a heat and/or shock (de)magnetization process (e.g., volcanoes or impact craters). Positive and negative correlations are used to argue for or against an active dynamo

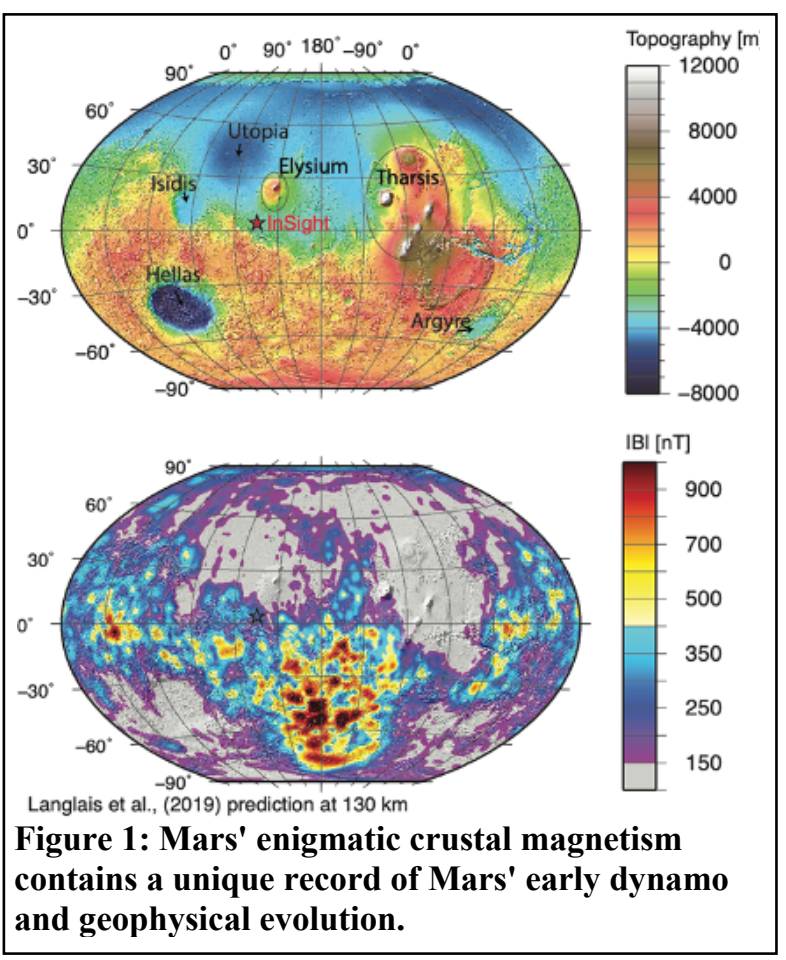
at the time at which the feature was emplaced (i.e., the feature either pre- or post-dated a dynamo cessation), allowing us to build up a geochronological timeline.

A prominent example is the very weak magnetic field intensity observed above some of the longlived volcanic provinces such as Tharsis and Elysium that likely thermally demagnetized previously magnetized buried volcanoes by reheating during later eruptions ${ }^{16,17}$. Furthermore, the general lack of magnetic field signatures above the large basins Hellas, Argyre, Isidis and Utopia has been used to argue for the absence of a global dynamo field at the time these impacts occurred. A dynamo that terminated before the basin-forming impacts occurred $(\sim>\mathrm{Ga})$ is consistent with the observations that most of the strong crustal fields are concentrated in areas of Noachian-aged surface terrain, and magnetic signatures associated with large craters ${ }^{3,18-21}$. However, the presence of non-negligible crustal fields above surface features such as volcanoes and lava plains that are younger than $4 \mathrm{Ga}^{22,23}$ suggest that the Martian dynamo could have operated later/longer. These interpretations are complicated by the presence of older, deeper magnetizable crust beneath these features that could be responsible for the measured fields. 
Recently and based on MAVEN data, it has been suggested that a dynamo could have operated before (at $4.5 \mathrm{Ga}$ ), after (at $3.7 \mathrm{Ga}$ ), and possibly during basin emplacement ${ }^{24}$.

Limitations: The spatial resolution of these models is limited to spatial scales comparable to the altitudes of orbital observations. Therefore, robust modeling of crustal magnetic fields globally is currently limited to spatial scales of $\sim 135 \mathrm{~km}$. The value of much higher resolution is amply demonstrated in the terrestrial case, where near-surface magnetic surveys detected the prominent north-south magnetized "stripes" along mid oceanic ridges. These stripes formed the primary supporting evidence for both plate tectonics and dynamo field reversals. Figure 2 shows that these features are not detectable from orbital altitudes ${ }^{25}$.

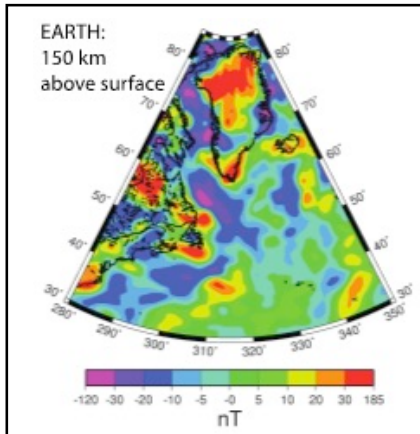

Figure 2: Evidence for geomagnetic reversals and plate tectonics cannot be seen at orbital altitudes.

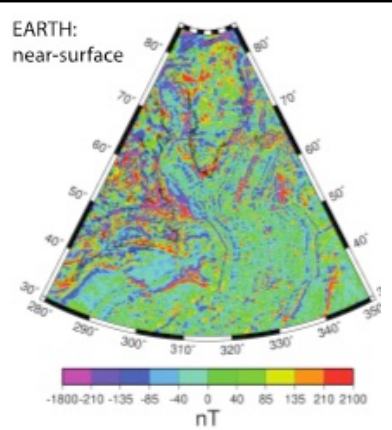

\section{Surface magnetic field data: The InSight} .

mission has provided the first magnetometer measurement on the martian surface and shows magnetic field amplitudes $>2000 \mathrm{nT}$, i.e. 10 times larger than those predicted from orbital data. This indicates the presence of magnetization at spatial scales significantly smaller than the $\sim 135$ $\mathrm{km}$ resolvable with MAVEN data and that are thus not observable from orbit ${ }^{26}$. Geological mapping of the InSight Landing site suggests that the magnetized layer is buried by between 200 $\mathrm{m}$ to $\sim 10 \mathrm{~km}$ of lava flows and modified material and is at least $3.9 \mathrm{Ga}^{26}$. Magnetization depths, and whether they are within the crust or the mantle, can be investigated if the absolute thickness of the crust (e.g., via InSight) is combined with high spatial resolution models for the magnetic field (like exist for the moon ${ }^{27}$ ).

Limitations: The depth and thickness of the magnetized layer are poorly known. InSight data represent a single data point for the surface crustal magnetic field and provide very limited information about its global distribution. Moreover, constraining the depth and thickness of the magnetized layer is only possible with much higher spatial resolution ${ }^{28}$.

Meteorites and information on magnetic carriers: To date, laboratory analyses of martian material have only been possible with meteorites. Magnetizations carried within the oldest known meteorite ALH 84001 suggest Earth-like paleointensities at $\sim 4 \mathrm{Ga}$, consistent with the hypothesis of an early dynamo ${ }^{29-32}$. A variety of potential magnetic carriers have been suggested including magnetite, hematite, titanohematite, titanomagnetite and pyrrhotite ${ }^{33}$. These vary in Curie/blocking temperatures, saturation magnetization and their ability to maintain their magnetization over long periods of time ${ }^{33,34}$. Further possible candidates are titanomagnetiteilmenite exsolution lamellae which have been shown to host strong magnetizations on Earth ${ }^{35}$. Constraining the magnetization depth (see previous section), and therefore whether magnetization lies within the crust or also the mantle, will help to constrain the source of magnetization, i.e., the magnetic minerals. Furthermore, while thermoremanence is typically assumed to be the main type of remanence ${ }^{22,36,37}$, shock ${ }^{38}$ and chemical ${ }^{14,39}$ remanent magnetization (CRM) might also have been important. CRM is particularly interesting because most CRM processes considered for Mars involve hydration reactions.

Limitations: Although radiometric dating allows for direct age determinations on meteorites (a major advantage over chronologies inferred from orbital measurements) the meteorite 
record can be difficult to interpret due to limited constraints on the provenance of the samples (i.e., where on Mars they came from) and their complex histories, including exposure to shock and multiple reheating events. This lack of context also contributes to uncertainty as to whether the magnetization was acquired in a crustal magnetic field or dynamo magnetic field.

Outstanding questions. Recent advances have provided insight into some aspects of the crustal magnetic field but have raised further questions. Although knowledge of the spatial structure of the crustal field has been significantly improved, we are still lacking a basic understanding of (1) the history and properties of the martian dynamo, (2) the source and nature of magnetic remanent magnetism and, (3) processes leading to observed magnetization.

Much of the evidence for what we believe is model-dependent, because we lack 'ground truth' for crustal magnetization, in terms of its strength, direction, age, or mineral carriers. The three major outstanding science questions are:

1) What were the characteristics of the martian dynamo?

a. When did the martian dynamo operate? Did it ever stop and restart?

b. What was its driving mechanism and did it change over time?

c. When did it cease permanently and how quickly did this happen?

d. What was the average surface field strength, and did it vary over time?

e. Was it dominantly dipolar or did it have substantial non-dipolar contributions? If dipolar, how closely was the dipole axis aligned with the rotation axis?

f. Is there evidence in the magnetic field record for major crustal reorganization (e.g. continental drift) during the period of operation of the dynamo?

g. Did the martian dynamo reverse polarity and, if so, how frequently and what were the characteristics of the reversals (duration, global field weakening)?

2) What is the nature of martian crustal remanent magnetism?

a. What are the major carriers of crustal magnetization?

b. How is the magnetization distributed in terms of depth and geography?

c. What is the global pattern of Mars' crustal magnetic fields at $\sim \mathrm{km}$ scales and how does it correlate with other characteristics such as topography, gravity, morphology and stratigraphy? Is there evidence for past plate tectonics?

d. How strong is the martian crustal magnetization and how does its strength vary under different geological conditions?

3) What are magnetization acquisition processes responsible for Mars' remarkably strong crustal magnetic fields?

a. Is thermoremanent magnetization the prevailing mechanism?

b. Could chemical or shock remanent magnetization be responsible for significant fractions of Mars' crustal magnetization?

c. What does the relative importance of thermal, chemical, or shock remanent magnetization in different geologic settings tell us about prevailing conditions that existed when the magnetization was acquired?

Lastly, we add a list of questions of importance for Future Exploration Efforts:

a. How do different crustal field regions affect the radiation environment at the surface?

b. What are implications of this distribution for astrobiology and human exploration?

c. Which areas could be of advantage with respect to potential resource identification, location, and exploration? 
These open questions motivate low altitude missions that can capture small-wavelength features and thus provide first-of-their-kind insight into local and regional magnetic fields including their amplitude and distribution, allowing improved correlations with geological features to improve constraints on dynamo timing.

Further characterization of magnetic properties will also greatly benefit from returned samples to be analyzed using state-of-the-art laboratory techniques. These will allow detailed magnetic analysis of a variety of samples, with known geological context and orientation.

\section{Recommendation for future measurements. We recommend that a} magnetometer be mounted on a (preferentially) mobile surface and/or low altitude aerial platform to allow spatially continuous measurements of crustal magnetic fields. In particular, a magnetometer mounted on a helicopter, aircraft or balloon will allow high resolution spatial coverage over spatial scales of tens to hundreds of kilometers, offering access to areas that might be hazardous or inaccessible from the surface.

Upcoming Opportunities. Planned sampling of the Mars 2020 rover will provide us with information on the $\mu \mathrm{m}-\mathrm{mm}$ scale. We recommend direct magnetic and radiometric analyses of (i) oriented Martian bedrock samples (i.e., samples whose original orientations are known) lacking complex metamorphic, aqueous alteration, and shock histories. Samples should preferably include a (ii) range of different ages ranging from the Early Noachian up to the Hesperian. The samples should not be exposed to (1) strong magnetic fields, (2) high temperature and (3) high pressures $\left(>200 \mu \mathrm{T},<100^{\circ} \mathrm{C},<0.1 \mathrm{GPa}\right)^{40}$. Main objectives from a paleomagnetics perspective were identified and ranked in a community-based study, discussed in more detail in ${ }^{40,41}$. Those address some of the outstanding questions particularly regarding carrier characteristics.

However, to put any of the samples into a global context we would like to make recommendations on future mission planning.

Table 1: Existing data and optimal platforms for important scales of crustal magnetic fields.

\begin{tabular}{|l|l|l|}
\hline Scales & Data and Studies so far & Optimal Measurement Platform \\
\hline Global; resolution of $\sim 150 \mathrm{~km}$ & MGS and MAVEN & Satellites \\
\hline$\sim 100 \mathrm{~s}-1000 \mathrm{~s} \mathrm{~km}$ & Missing & Airplanes, Balloons \\
\hline$\sim 1 \mathrm{~km}$ & Missing & Drone \\
\hline$\sim$ meters & Missing & Field Surveys, Rovers \\
\hline in-situ spot measurement & InSight & Lander \\
\hline$\mu \mathrm{m}-\mathrm{mm}$ & $\begin{array}{l}\text { (1) To some degree via meteorites } \\
\text { (2) Returned sample }\end{array}$ & Laboratory studies \\
\hline
\end{tabular}

We present the following possibilities of regional scale mapping with magnetometers with preference of the first two covering 100s-1000s of km:

- Balloons offer the opportunity to cover 100s-1000s of $\mathbf{k m}$ and spend a prolonged (months to years) time at a few $\mathrm{km}$ altitude, where they naturally rise during the day and set down at nighttime. Achievable resolution would provide magnetic field studies with unprecedented resolution allowing to correlate large areas and geological constructs such as craters, lava

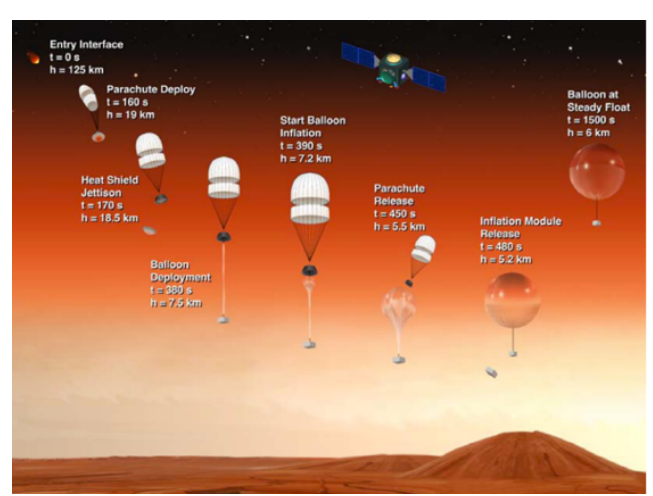

Figure 3: several concepts exist for Mars balloons 
flows, etc., with magnetic signals. Low atmospheric density on Mars presents a challenge and lightweight technology is required. Although concept studies have explored such designs, as well as deployment, inflation and separation of a Mars balloon ${ }^{42,43}$, these investigations have more recently been paused. Thus, we suggest that such studies should be continued and integrated in a future Mars mission.

- Airplanes such as the formerly proposed Aerial Regional-scale Environmental Survey (ARES) Mars scout airplane can cover 100s-1000s of $\mathrm{km}$ from 1-2 km altitude while flying a precision groundtrack. A mature mission and low risk flight system concept has been provided by earlier efforts ${ }^{44}$ and was deemed an appropriate mobile surface for regional studies ${ }^{44,45}$. Airplanes offer an alternative to balloon studies providing similar resolution, although a limited survey duration and thus science return.

- Drones/Helicopters in combination with mobile surfaces such as a rover offer a way to explore $1-10 \mathbf{~ k m} / \mathbf{s o l}$ around the rover with flight times $<10$ minutes. This would allow to characterize the surroundings in terms of stratigraphy, chemistry and other geological and geophysical properties in combination with and depending on other experiments on the rover. Another possibility (although less desirable because of the more limited range and magnetic cleanliness concerns) would be to add a magnetometer on the rover itself. Additionally, a susceptometer could be added for in situ measurements of the complex magnetic susceptibility at different frequencies. The instrument can be used to discern between rocks with the same chemical composition but from different origins and / or evolutions and to constrain the material conductivity. Such measurements would thus help determine the concentration of magnetic carriers and their organization in domains.

- Landers provide data points in different areas and allow to explore (a) the field strength at the surface and (b) the way external fields interact with it ${ }^{26}$. While the InSight magnetometer was not added as a science instrument it is enabling us to explore magnetic and space weather conditions at the surface which is essential for future human exploration missions. Given the scientific return of InSight's magnetometer so far, we strongly suggest that magnetometers are routinely added in future lander missions.

Importance for Space Exploration. Magnetic field variations are a proxy for subsurface geologic variations and, because of association of iron-bearing minerals with resources, are used for exploration of mineral resources. In space exploration, this also extends to identification and characterization of resources and building materials. Low altitude aerial magnetic surveys are needed for reconnaissance studies of target areas for mineral/ore exploration ${ }^{46}$. Decades of terrestrial experience in magnetic surveys will enable us to:

1) Characterize contrasts in Mars' crustal magnetization down to $\mathrm{km}$ and sub-km scales.

2) Evaluate resource potential by estimating the depth, volume, thickness, and magnetization of the magnetized crust. This will allow estimation of Fe content in the subsurface and identification and characterization of range of resources needed for human exploration from building materials to ore deposits.

3) Evaluate the potential of strong field regions for shielding the surface (and hence life and human explorers) from cosmic rays and solar energetic particles.

4) Establish improved context for in-situ by characterizing the crust at scales intermediate between those of surface operations and satellite data. 


\section{References}

1. Varney, R. H. et al. Journal of Geophysical Research : Space Physics Influence of ion outflow in coupled geospace simulations : 1 . Physics-based ion outflow model development. 9671-9687 (2016)

doi:10.1002/2016JA022778.Key.

2. Luhmann, J. G. et al. The intrinsic magnetic field and solar-wind interaction of Mars. in In: Mars (ed. George, M.) 1090-1134 (1992).

3. Acuna, M. H. et al. Global Distribution of Crustal Magnetization Discovered by the Mars Global Surveyor MAG/ER Experiment. Science (80-. ). 284, 790-793 (1999).

4. Acuña, M. H. et al. Magnetic field of Mars: Summary of results from the aerobraking and mapping orbits. $J$. Geophys. Res. 106, 23403 (2001).

5. Cain, J. C. An $\mathrm{n}=90$ internal potential function of the Martian crustal magnetic field. J. Geophys. Res. 108, 119 (2003).

6. Langlais, B. et al. Crustal magnetic field of Mars. J. Geophys. Res. 109, 1-16 (2004).

7. Morschhauser, A. et al. A spherical harmonic model of the lithospheric magnetic field of Mars. J. Geophys. Res. Planets 119, 1162-1188 (2014).

8. Breus, T. K. et al. The effects of crustal magnetic fields and the pressure balance in the high latitude ionosphere/atmosphere at Mars. Adv. Sp. Res. 36, 20432048 (2005)

9. Lillis, R. J. et al. Electron reflectometry in the martian atmosphere. Icarus 194, 544-561 (2008).

10. Connerney, J. E. P. et al. First results of the MAVEN magnetic field investigation. Geophys. Res. Lett. 42, 8819- 8827 (2015)

11. Mittelholz, A. et al. A New Magnetic Field Activity Proxy for Mars From MAVEN Data. Geophys. Res. Lett. 45, 5899-5907 (2018).

12. Langlais, B. et al. A New Model of the Crustal Magnetic Field of Mars Using MGS and MAVEN. J. Geophys. Res. Planet 124, 1542-1569 (2019).

13. Stanley, S. et al. Mars' paleomagnetic field as the result of a single-hemisphere dynamo. Science 321, 1822-1825 (2008).

14. Quesnel, Y. et al. Serpentinization of the martian crust during Noachian. Earth Planet. Sci. Lett. 277, 184-193 (2009).

15. Neumann, G. A. et al. Crustal structure of Mars from gravity and topography. J. Geophys. Res. E Planets 109, 1-18 (2004).

16. Johnson, C. L. et al. Evolution of the Tharsis region of Mars: Insights from magnetic field observations. Earth Planet. Sci. Lett. 230, 241-254 (2005).

17. Lillis, R. J. et al. Demagnetization of crust by magmatic intrusion near the Arsia Mons volcano: Magnetic and thermal implications for the development of the Tharsis province, Mars. J. Volcanol. Geotherm. Res. 185, 123138 (2009).

18. Lillis, R. J. et al. Time history of the Martian dynamo from crater magnetic field analysis. J. Geophys. Res. E Planets 118, 1488-1511 (2013).

19. Vervelidou, F. et al. Constraining the Date of the Martian Dynamo Shutdown by Means of Crater Magnetization Signatures. J. Geophys. Res. Planets 122, 2294-2311 (2017).

20. Arkani-Hamed, J. Timing of the Martian core dynamo. J. Geophys. Res. 109, 1-12 (2004).

21. Lillis, R. J. et al. Rapid decrease in Martian crustal magnetization in the Noachian era: Implications for the dynamo and climate of early Mars. Geophys. Res. Lett. 35, L14203 (2008).

22. Hood, L. L. et al. Magnetic anomalies near Apollinaris Patera and the Medusae Fossae Formation in Lucus Planum, Mars. Icarus 208, 118-131 (2010)

23. Schubert, G. et al. Geophysics: Timing of the Martian dynamo. Nature 408, 666-667 (2000).

24. Mittelholz, A. et al. Timing of the martian dynamo : New constraints for a core field 4.5 and $3.7 \mathrm{Ga}$ ago. $S c i$. Adv. 18, 1-8 (2020).

25. Ravat, D. Interpretation of Mars southern highlands high amplitude magnetic field with total gradient and fractal source modeling: New insights into the magnetic mystery of Mars. Icarus 214, 400-412 (2011).

26. Johnson, C. L. et al. Crustal and time-varying magnetic fields at the InSight landing site on Mars. Nat. Geosci. 13, (2020).

27. Wieczorek, M. A. Strength, Depth, and Geometry of Magnetic Sources in the Crust of the Moon From Localized Power Spectrum Analysis. J. Geophys. Res. Planets 123, 291-316 (2018)

28. Vervelidou, F. et al. Global maps of the magnetic thickness and magnetization of the Earth's lithosphere. Earth, Planets Sp. 67, (2015).

29. Weiss, B. P. et al. Records of an ancient Martian magnetic field in ALH84001. Earth Planet. Sci. Lett. 201, 449-463 (2002).

30. Weiss, B. P. et al. Paleointensity of the ancient Martian magnetic field. Geophys. Res. Lett. 35, 1-5 (2008).

31. Gattacceca, J. et al. Martian meteorites and Martian magnetic anomalies: A new perspective from NWA 7034. Geophys. Res. Lett. 41, 4859-4864 (2014).

32. Rochette, P. et al. Pyrrhotite and the remanent magnetization of SNC meteorites: A changing perspective on Martian magnetism. Earth Planet. Sci. Lett. 190, 1-12 (2001).

33. Dunlop, D. J. et al. Magnetic minerals in the Martian crust. J. Geophys. Res. E Planets 110, 1-11 (2005).

34. Rochette, P. et al. Matching Martian crustal magnetization and magnetic properties of Martian meteorites. Meteorit. Planet. Sci. 40, 529 (2005).

35. McEnroe, S. A. et al. Magnetic anomalies, layered intrusions and Mars. Geophys. Res. Lett. 31, 31-34 (2004).

36. Bowles, J. A. et al. Magnetic and petrologic characterization of synthetic Martian basalts and implications for the surface magnetization of Mars. $J$. Geophys. Res. E Planets 114, 1-18 (2009).

37. Milbury, C. et al. Search for the global signature of the Martian dynamo. J. Geophys. Res. 115, E10010 (2010).

38. Hood, L. L. Distribution of crustal magnetic fields on Mars: Shock effects of basin-forming impacts. Geophys. Res. Lett. 30, 1-4 (2003).

39. Harrison, K. P. et al. Groundwater-controlled valley networks and the decline of surface runoff on early Mars. J. Geophys. Res. E Planets 110, 1-17 (2005).

40. Weiss, B. P. et al. Paleomagnetic Studies of Returned Samples from Mars. 2nd Int. Mars Sample Return 2018 (2018).

41. Mittelholz, A. et al. The Mars 2020 Candidate Landing Sites: A Magnetic Field Perspective. Earth Sp. Sci. 5, 410-424 (2018).

42. Hall, J. L. et al. Flight test results for aerially deployed mars balloons. AIAA Balloon Syst. Conf. 2007 262-274 (2007).

43. Kerzhanovich, V. V. et al. Breakthrough in Mars balloon technology. Adv. Sp. Res. 33, 1836-1841 (2004).

44. Braun, R. D. et al. Design of the ARES Mars airplane and mission architecture. J. Spacecr. Rockets 43, 10261034 (2006).

45. Wright, H. et al. ARES Mission Overview - Capabilities and Requirements of the Robotic Aerial Platform. in 2nd AIAA 'Unmanned Unlimited' Conf. and Workshop \&amp; Exhibit (2003). doi:10.2514/6.2003-6577.

46. Meinert. L. D., Space Resources - A Framework for the Future. Soc. Econ. Geol. Newsl. 117, 14-20 (2019). 